\title{
Method for estimating non-study cigarette use among switchers to low nicotine content cigarettes in ambulatory clinical studies
}

Mingda Zhang ( $\square$ mingda.zhang@altria.com )

Altria Client Services https://orcid.org/0000-0001-8138-2722

Jeffery Edmiston

Altria Client Services

George Karles

Altria Client Services

Donna Smith

Altria Client Services

Research article

Keywords: Clinical study, protocol deviation, noncompliance, low nicotine cigarette, VLN, biomarker

Posted Date: September 24th, 2019

DOI: https://doi.org/10.21203/rs.2.14909/v1

License: (1) This work is licensed under a Creative Commons Attribution 4.0 International License.

Read Full License

Version of Record: A version of this preprint was published at International Journal of Clinical Research \& Trials on January 20th, 2020. See the published version at https://doi.org/10.15344/2456-

8007/2020/141. 


\section{Abstract}

Background FDA is considering to establish a product standard to reduce nicotine in cigarettes to make them "minimally addictive or nonaddictive." FDA has funded many clinical studies where smokers are switched to smoking low nicotine cigarettes to determine a nicotine ceiling that is appropriate for the protection of the public health. Unlike typical clinical trials involving pharmaceuticals or medical devices, ambulatory studies with low nicotine cigarettes face a unique challenge in that conventional nicotine non-study cigarettes are readily available to participants when protocols require them to exclusively use study cigarettes. As a consequence, protocol deviation in non-study product use is a major limitation common in such ambulatory studies, with up to 80 percent of participants using non-study cigarettes during the study. There is no published method for estimating the magnitude of such protocol deviation, i.e., the number of non-study cigarettes smoked by participants, in such studies.

Methods We present a method for estimating the magnitude of noncompliance based on the proposition that the level of biomarker of exposure to a smoke constituent is proportional to the amount of the constituent per cigarette and the number of cigarettes smoked by participants. The method estimates the number of non-study cigarettes smoked by participants based on the discrepancies between the yield of smoke constituents (e.g., nicotine) and the level of the corresponding biomarkers measured in a study.

Results Data from a confined study confirmed the validity of this method. Under-reporting on the magnitude of non-study cigarette use is widespread across studies using different low nicotine cigarettes. Participants in one of the largest published studies under-reported the number of non-study cigarette used by $79-90 \%$.

Conclusions Controlling and accurately estimating non-study cigarette use is critical for ambulatory low nicotine cigarette switching studies to ensure the resulting data can be appropriately evaluated to support science-based regulatory decision-making. In planning future studies, researchers should consider incorporating specific biomarkers that would enable objective assessment of both the prevalence and the magnitude of non-study cigarette use.

\section{Background}

The US FDA issued an advance notice of proposed rulemaking (ANPRM) in 2018 entitled "Tobacco Product Standard for Nicotine Level of Combusted Cigarettes" [1] indicating that it is considering the issuance of a product standard to set a maximum nicotine level in cigarettes so "they are minimally addictive or nonaddictive, using the best available science to determine a level that is appropriate for the protection of the public health". SPECTRUM ${ }^{\circledR}$ research cigarettes, with up to $98 \%$ less nicotine than conventional commercial cigarettes, have been widely used in clinical studies on very low nicotine (VLN) cigarettes. Among the dozens of 
clinical studies using the SPECTRUM ${ }^{\circledR}$ VLN cigarettes registered at clinicaltrials.gov, the overwhelming majority are of ambulatory design. Ambulatory studies with low nicotine cigarettes face a unique challenge not typically encountered in clinical trials involving pharmaceuticals and medical devices in that conventional non-study cigarettes with up to 50fold-higher nicotine content than the study cigarettes are readily accessible to participants. When studies are designed to test the effect of exclusive use of the low nicotine cigarette, the use of conventional nicotine content (CNC) non-study cigarettes makes it difficult to interpret the results. In fact, protocol deviation in non-study product use is a major limitation commonly observed in studies where smokers are assigned to exclusively smoke VLN cigarettes. As an example, in the largest immediate nicotine reduction switching study with 840 randomized participants [2], 73-81\% of the participants assigned cigarettes with nicotine content of 5.2 $\mathrm{mg} / \mathrm{g}$ tobacco or less self-reported smoking at least one non-study cigarette throughout the duration of the study. In addition, based on the observed levels of urinary cotinine, a biomarker of exposure (BOE) to nicotine, the authors showed that study participants substantially under-reported the prevalence of non-study cigarette use [3, 4]. During week 6 of the study alone, $39 \%$ of participants assigned to cigarettes with $0.4 \mathrm{mg} / \mathrm{g}$ nicotine (about $98 \%$ lower than $\mathrm{CNC}$ ) self-reported smoking non-study cigarettes, whereas urinary cotinine assessment showed that 76-78\% of them were non-compliant [4]. The difference between the expected and observed levels of urinary total nicotine equivalents (nicotine plus its major metabolites, TNE) also provides an indication on the degree of under-reporting of the magnitude of non-study cigarette use (i.e., number of cigarettes smoked per day [CPD]). The urinary TNE levels for the study group assigned the $0.4 \mathrm{mg} / \mathrm{g}$ VLN cigarettes were expected to be $0.5 \mathrm{nmol} / \mathrm{mg}$ creatinine if the participants were compliant by exclusively smoking the assigned study cigarettes [5]. However, the actual TNE level was reported to be 30 times higher at $15 \mathrm{nmol} / \mathrm{mg}$ creatinine [2]. The most plausible explanation is that these participants supplemented the study cigarettes with non-study cigarettes containing up to 50 times more nicotine, likely their usual brand (UB) cigarettes [5].

Such high levels of under-reporting of non-study cigarette use, in both the prevalence and the magnitude, can significantly influence study outcome measures and pose major challenges to accurately interpreting the resulting data. While there have been published methods for estimating the prevalence (i.e., percent of participants) of such noncompliance [3, $4,6]$, no method for estimating its magnitude (i.e., number of CPD) has been published. We 
present a method for estimating the magnitude of non-study cigarette use among switchers to VLN cigarettes that is based on the same proposition used in the published methods for biochemically estimating the prevalence of noncompliance $[3,4,6]$. We also discuss the implication of the resulting estimates on the interpretation of data from ambulatory VLN cigarette switching studies.

\section{Methods}

Based on the proposition that, if study participants use the assigned study cigarettes exclusively (per protocol), the level of the BOE to a smoke constituent should be proportional to the quantity of the constituent per cigarette (in smoke or tobacco, referred to as "Yield" for simplicity) and the number of cigarettes smoked per day (CPD):

$$
\mathrm{BOE}=\text { Yield } * \mathrm{CPD} * \mathrm{k}
$$

$\mathrm{k}$ represents a composite factor comprising how a cigarette is smoked and the distribution and clearance of the constituent.

When study participants smoke non-study cigarettes in addition to the assigned VLN study cigarettes, this equation needs to be modified to account for the contributions from both sources:

$$
\mathrm{BOE}=\text { Yield }_{\mathrm{VLNC}}{ }^{*} \mathrm{CPD}_{\mathrm{VLNC}} * \mathrm{k}_{\mathrm{VLN}}+\text { Yield }_{\text {non-study }}{ }^{*} \mathrm{CPD}_{\text {non-study }}{ }^{*} \mathrm{k}_{\text {non-study }}
$$

The $\mathrm{k}$ value for each constituent/biomarker combination depends primarily on the actual intake relative to the constituent Yield per cigarette (referred to as "bioavailability" for consistency with existing literature $[3,6,7]$ ) and the distribution and clearance of the constituent. Within the same individual or between randomized groups of participants, for which interindividual pharmacokinetic variations are controlled, the difference in the $\mathrm{k}$ value between study and nonstudy cigarettes is mainly driven by the bioavailability of the constituent, with compensatory smoking behavior (i.e., puffing and inhalation) being the primary factor. Based on smoking topography and exhaled CO measures, published VLN cigarette clinical studies generally reported no or limited compensatory smoking behaviors between VLNC, CNC and UB cigarettes [2, 8-10]. Therefore, the $\mathrm{k}$ values for study and non-study cigarettes can be expected to be similar and are assumed to be the same to simplify the equation to:

$$
\mathrm{BOE}=\text { Yield }_{\mathrm{VLNC}}{ }^{*} \mathrm{CPD}_{\mathrm{VLNC}} * \mathrm{k}+\text { Yield }_{\text {non-study }}{ }^{*} \mathrm{CPD}_{\text {non-study }}{ }^{* \mathrm{k}}
$$


While it is possible that some study participants could have smoked non-commercial cigarettes or used other tobacco products, VLN cigarette studies typically exclude users of such products $[2,11]$. The most likely non-study cigarettes smoked by the participants are the conventional commercial UB cigarettes they have been smoking prior to enrolling in the study [5, 12]. Study participants smoke many different cigarette brands as their UB cigarettes, making precise determination of the average constituent yields for UB cigarettes impractical. SPECTRUM ${ }^{\circledR}$ cigarettes, manufactured by the $22^{\text {nd }}$ Century Group, Inc. and distributed by the National Institute on Drug Abuse (NIDA) [13], have been adopted as standard research

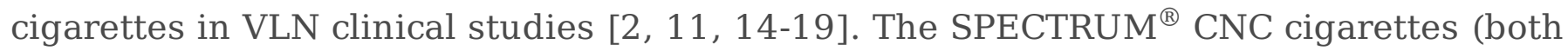
menthol and non-menthol), with a nominal nicotine content of $15.8 \mathrm{mg} / \mathrm{g}$ tobacco were designed to be representative of the average commercial cigarettes in the US market and are commonly used as CNC cigarette references in clinical studies. Therefore, the constituent Yields of the SPECTRUM ${ }^{\circledR}$ CNC cigarettes can be used as surrogates for the average yields of the non-study cigarettes in the equation:

$$
\mathrm{BOE}=\text { Yield }_{\mathrm{VLNC}} * \mathrm{CPD}_{\mathrm{VLNC}} * \mathrm{k}+\text { Yield }_{\mathrm{CNC}} * \mathrm{CPD}_{\text {non-study }}{ }^{*} \mathrm{k}
$$

The number of non-study cigarettes smoked by participants in a VLN cigarette group can then be calculated as:

$$
\mathrm{CPD}_{\text {non-study }}=\left(\mathrm{BOE}-\text { Yield }_{\mathrm{VLNC}}{ }^{*} \mathrm{CPD}_{\mathrm{VLNC}} * \mathrm{k}\right) /\left(\text { Yield }_{\mathrm{CNC}} * \mathrm{k}\right)
$$

The $\mathrm{k}$ value can be derived using data from the SPECTRUM ${ }^{\circledR}$ CNC cigarette group, based on the equation $\mathrm{BOE}=$ Yield ${ }^{*} \mathrm{CPD} * \mathrm{k}$, as:

$$
\mathrm{k}=\mathrm{BOE}_{\mathrm{CNC}} /\left(\operatorname{Yield}_{\mathrm{CNC}} * \mathrm{CPD}_{\mathrm{CNC}}\right)
$$

Therefore, the magnitude of non-study cigarette use among VLN cigarette groups $\left(\mathrm{CPD}_{\text {non- }}\right.$ study) can be estimated using equation [5] for studies when BOE, Yield, and CPD data are available for both the VLN and CNC (or UB) cigarette groups.

\section{Results}

\section{Method Verification}

The validity of the proposed method can be assessed with data from VLN cigarette clinical studies in which the number of study cigarette smoked by the participants are fully verified. There is one published VLN cigarette switching study where participants were fully compliant 
with study product use because they were confined in a hotel and did not have access to nonstudy cigarettes [12]. Denlinger et al. reported that, after 4 days of exclusive use of the 0.4 $\mathrm{mg} / \mathrm{g}$ nicotine cigarettes, the average within-subject reductions for urinary cotinine, TNE, and anatabine (a minor tobacco alkaloid) were 92\%, 94\%, and 93\%, respectively, compared to baseline when the participants were smoking their UB cigarettes. In contrast to the results from Donny et al. [2] discussed earlier, these reductions closely reflect the relative reduction in nicotine and anatabine contents (98\% and 97\%, respectively) between the $0.4 \mathrm{mg} / \mathrm{g}$ VLN cigarettes and UB cigarettes [12]. Taking into consideration the $40 \%$ increase in mean CPD from baseline, the authors postulated that the discrepancy between the percentage reductions in nicotine or anatabine content in the cigarettes and their corresponding biomarkers could be accounted for by the carryover of the biomarkers from the baseline prior to the participants entering the study.

To test the validity of our method, we calculated non-study cigarette estimates using the data reported in Denlinger et al. [12] (Table 1). The estimated numbers of non-study CPD using our method are 0.3, 0.6, 0.9 and 0.4 , based on the reported levels of urinary nicotine, TNE, cotinine and anatabine, respectively (Table 1), all within 1-3\% of the actual average CPD smoked by the participants (28.3). The highest estimate (0.9 CPD) was from cotinine which has the longest half-life (16-19 hours) [20] whereas the lowest (0.3 CPD) was from nicotine which has the shortest half-life (about 2 hours) [21] among the four biomarkers, suggesting these small discrepancies between the estimated and the actual CPD numbers likely reflect the carryover of the baseline biomarkers from UB cigarettes as the authors concluded [12]. These results provide evidence supporting the validity of the proposed method and the assumptions regarding the $\mathrm{k}$ factor and UB constituent Yields used in the method.

Table 1. Estimate of Non-study CPD with Data from Denlinger et al.[12]

\begin{tabular}{|c|c|c|c|}
\hline \multicolumn{3}{|c|}{ Data from Denlinger et al. } & \\
\hline Cigarette & VLNC $(0.4 \mathrm{mg} / \mathrm{g})$ & UB & \\
\hline \multicolumn{3}{|c|}{ Yield (per gram of tobacco filler) } & \\
\hline Nicotine (mg) & 0.295 & 16.5 & \\
\hline Anatabine $(\mu \mathrm{g})$ & 30 & $1158.5^{*}$ & \\
\hline CPD & 28.3 & 20.09 & \\
\hline \multicolumn{3}{|c|}{$\begin{array}{l}\text { BOE } \\
\text { (nmol/mg creatinine) }\end{array}$} & Non-study CPD Estimate \\
\hline Nicotine & 0.47 & 11.47 & 0.3 \\
\hline TNE & 3.45 & 63.38 & 0.6 \\
\hline Cotinine & 1.23 & 17.79 & 0.9 \\
\hline Anatabine & 0.003 & 0.06 & 0.4 \\
\hline
\end{tabular}




\section{Magnitude of Under-reporting at Different Nicotine Levels}

We applied our method to data from the study discussed in the Background section [2], the largest double-blind, parallel group, ambulatory clinical trial in which participants were randomly assigned to exclusively smoke SPECTRUM ${ }^{\circledR}$ cigarettes at one of five nicotine levels: from 0.4 to $15.8 \mathrm{mg} / \mathrm{g}$ tobacco filler or their UB cigarettes for 6 weeks. While the prevalence of noncompliance was reported to be high by the authors, as discussed earlier, the average numbers of self-reported non-study CPD were much lower $\left(75^{\text {th }}\right.$ percentile $\leq 2$ cigarettes for all groups during week six) $[2,4]$ than would be expected from the levels of urinary BOEs.

With the method described here, data reported in this study allow eighteen separate non-study CPD estimates for each of the five VLN groups using different combinations of BOE, Yield, and CPD parameters (Table S1) using the $15.8 \mathrm{mg} / \mathrm{g}$ CNC group as the reference. Using data reported for week six (Table S2), on which the primary outcome of the study were based, the average estimates of non-study CPD based on the two BOEs for nicotine (cotinine and TNE) are fairly close to each other for each group whereas estimates based on 4(methylnitrosamino)-1-(3-pyridyl)-1-butanol [NNAL, a biomarker for 4-(methylnitrosamino)-1-(3pyridyl)-1-butanone (NNK)] tend to be higher, particularly for the groups assigned cigarettes with $0.4 \mathrm{mg} / \mathrm{g}$ nicotine content (Figure 1).

Comparison between the estimated average non-study CPD in Figure 1 with the numbers self-reported by study participants for each VLN cigarette group shows that participants under-reported non-study CPD by 73-89\% (Figure 2). In addition, the under-reported CPD numbers (7.1 to 10.5) are higher than the corresponding self-reported CPD reductions (4.8 to 6.4) after switching to cigarettes with nicotine levels of $2.4 \mathrm{mg} / \mathrm{g}$ or less for six weeks (Figure 3). When these under-reported non-study CPD numbers are taken into consideration, there was no reduction in the estimated total CPD (primary outcome of the study) for each VLN group, compared to the CNC reference group (Figure 3).

Repeating the analyses using the UB group as the reference, assuming comparable nicotine and NNK levels between UB cigarettes and the $15.8 \mathrm{mg} / \mathrm{g}$ CNC cigarettes, produced non-study CPD estimates that were about $10 \%$ higher than those using the $15.8 \mathrm{mg} / \mathrm{g} \mathrm{CNC}$ cigarettes as the reference (Figure S1). Estimates using the UB group as the reference are 
likely more accurate than those with $15.8 \mathrm{mg} / \mathrm{g}$ CNC cigarettes as the reference because the participants should be more compliant with reference product use.

\section{Magnitude of Under-reporting Across Different Studies}

To investigate whether under-reporting non-study cigarette use is common in different VLN cigarette switching studies, we applied this method to data from one study using cigarettes manufactured by Philip Morris USA [22] and two studies using cigarettes from the Vector Group $[9,23]$. Figure 4 presents the resulting average non-study CPD estimates and shows that there was under-reporting of non-study cigarette CPD across all three studies, although at lower magnitude than in Donny et al. [2]. Similar to the finding with data from Donny et al. [2] in the previous section, the estimated end of study total CPD are higher than baseline CPD for each VLN cigarette group in all three studies.

A more recent clinical study compared the effects of immediate reduction to $0.4 \mathrm{mg} / \mathrm{g}$ nicotine SPECTRUM ${ }^{\circledR}$ cigarette versus gradual nicotine reduction (from 15.5 to 11.7, 5.2, 2.4, and then to $0.4 \mathrm{mg} / \mathrm{g}$ over 20 weeks) on BOE [11]. Using International Organization of Standardization (ISO) smoke nicotine and NNK yields and the corresponding reported mean TNE and NNAL levels, our method generated an estimated average of 8.6 non-study CPD for the immediate reduction group in the study. This is close to the 9.6 non-study CPD estimated for the group assigned to the same VLN (0.4 mg/g) cigarette in Donny et al. [2] (Figure S1), with UB cigarettes as the reference in both cases. Estimation of non-study CPD for the gradual reduction group in this study was not possible due to how the data were reported in the publication [11] and unavailability of the raw data for analysis.

Combined, the results of the analyses using our method indicate that under-reporting on the magnitude of non-study cigarette use is common across switching studies involving different VLN cigarettes.

\section{Discussion}

Assessing study participants' compliance with study cigarette use is critical to quantifying the effects of switching to VLN cigarettes [24]. Previously reported high protocol deviation prevalence rates in non-study cigarettes use based on both self-report and biochemical thresholds [3, 4, 6] are likely driven by a combination of the reduction in nicotine and sensory dissatisfaction with the SPECTRUM ${ }^{\circledR}$ research cigarettes used [4]. We present a 
method for estimating the magnitude of non-study cigarette use in ambulatory VLN cigarette switching studies where participants continue to have access to CNC cigarettes. Data from a published study in which the participants were known to be using study cigarettes exclusively [12] confirmed the validity of this method. Applying this method to data from five switching studies of various durations using different VLN cigarettes, and including both immediate and gradual nicotine reduction approach $[2,9-11,22]$, showed that under-reporting on the magnitude of non-study cigarette use is common across studies, and that when underreporting is taken into consideration, self-reported CPD reductions in these studies disappear.

Given that the nicotine content is expected to be well characterized for VLN cigarettes used in switching studies and the urinary cotinine level is likely the most frequently measured biomarker in such studies, this combination is a straightforward way to estimate non-study CPD. Using TNE instead of cotinine would mitigate the impact of interindividual variability in nicotine metabolism and enhance the accuracy of the resulting estimate. Data from studies using SPECTRUM ${ }^{\circledR}$ cigarettes are the most suitable for estimating non-study CPD because these cigarettes have been well characterized both physically and chemically $[25,26]$.

One limitation of this paper is that we estimated some CPD and biomarker values from graphs in the cited publications when the actual numbers were not reported. Comparisons between the estimated and reported actual values, where available, indicate that the estimates from the graphs closely match the actual values. Another limitation of this paper is the use of "average" values for each group as presented in the respective publications that use different statistical models to calculate and adjust for the group averages. Repeating the calculations using raw data for individual participants could address both limitations; however, these data are not published or publicly available.

The method used here to estimate the amount of CNC non-compliance in ambulatory VLN cigarette studies assumes that the VLN cigarettes and the CNC cigarettes are smoked similarly. If the VLN cigarettes are smoked significantly more intensely, the bioavailability for each cigarette type would be different. Benowitz et al. [3, 24] assessed non-compliance thresholds and made an allowance for a theoretical 4-fold increase in nicotine bioavailability to account for potentially more intense compensatory smoking of VLN cigarettes (i.e., assuming a $40 \%$ nicotine bioavailability for VLN cigarettes as compared to $10 \%$ for UB cigarettes). If true, such differential compensatory smoking would result in overestimation of non-study CPD numbers using the method presented here because the bioavailability, and hence the k values, 
would be different for VLN and CNC cigarettes under such conditions. However, data from the controlled VLN cigarette study with fully compliant participants led Denlinger et al. [12] to conclude that the cutoff assuming a 4-fold increase in bioavailability of nicotine is overly conservative. In fact, VLN cigarette studies were more likely to report compensatory smoking in the opposite direction, i.e., the total puff volume per cigarette tends to be lower for VLN cigarettes compared to UB cigarettes $[2,9,10]$. Therefore, our assumption for similar $\mathrm{k}$ values between VLN and CNC cigarettes is unlikely to result in over-estimation of the magnitude of noncompliance. On the contrary, earlier publications that included the allowance for a 4-fold increase in nicotine bioavailability for VLN cigarettes in setting the noncompliance thresholds likely have underestimated the prevalence of non-study cigarette use $[3,4,6]$.

As discussed previously, compensatory smoking can affect non-study CPD estimates using this method. However, due to the limited amount of nicotine in the tobacco of the lowest VLN cigarettes, the impact of compensatory smoking on the non-study CPD estimates is expected to be minimal. For example, assuming 40\% nicotine bioavailability [24] (equivalent to approximately 3 times the amount under Canadian Intense smoking conditions) would reduce the non-study CPD estimate for the $0.4 \mathrm{mg} / \mathrm{g}$ group in Donny et al. [2] by less than 1 cigarette (or about 10\%). Compensatory smoking is more likely to cause overestimation with this method among groups switching to cigarettes at higher nicotine levels. Therefore, caution is warranted when applying this method to data from studies in which substantial compensatory smoking is observed among groups smoking VLN cigarettes, particularly those with intermediate levels of nicotine content (e.g., above $5.2 \mathrm{mg} / \mathrm{g}$ ). Further, this method should not be used for studies with conventional cigarettes with low machine smoke nicotine yields (but similar nicotine contents in tobacco), where smokers' actual nicotine intake has been shown to have lower proportionality to both the machine smoking nicotine yield of the cigarettes [27] and the number of cigarettes smoked per day [28].

While high level of environment tobacco smoke (ETS) exposure could impact the biochemical threshold for estimating the prevalence of noncompliance [4, 6], among groups smoking the lowest nicotine content cigarettes, ETS is not likely to have a major impact on estimating the magnitude of non-study cigarette use because even under exceptionally heavy ETS exposure, the nicotine intake from ETS was still less than that from actively smoking one cigarette [29, 30]. 
BOEs for nicotine exposure will not be suitable when applying this method to studies in which other nicotine-containing products (e.g., nicotine replacement therapies, electronic nicotine devices) are expected to be used by the participants in addition to the VLN cigarettes, e.g., when alternative nicotine-containing products are explicitly included among the interventions [31] or the enrolled participants are known to use such products in addition to commercial cigarettes (i.e., dual/poly users). For such studies, BOEs to other smoke constituents that are either not present or present at much lower levels in the alternative nicotine products (e.g., NNK, anatabine) should be considered for inclusion to enable estimation of non-study cigarette use. Constituents that are tobacco-specific or to which exposure from other sources (e.g., food or air) is expected to be significantly lower than smoking are preferable for more reliable estimates. Similarly, BOEs for neither nicotine nor NNK can be used with this method in VLN cigarette studies where dual/poly use with moist smokeless tobacco product (MST) is anticipated among the study participants, due to the presence of high levels of both nicotine and NNK in these products.

Estimates using this method based on NNK tend to be higher and show larger variation than those based on nicotine, whereas estimates based on anatabine closely track those using nicotine. This observation, albeit based only on data from one study [12], suggests that anatabine has the potential to be superior to NNK for non-study CPD estimation in studies where nicotine cannot be used for this purpose. In shorter-term studies, anatabine has the added benefit of a shorter half-life than NNAL.

\section{Conclusions}

We present a method for estimating the magnitude of non-study product use in ambulatory VLN cigarette switching studies where participants continue to have access to non-study cigarettes. Results from our study indicate that, while the estimated number of non-study CPD vary by study, under-reporting the magnitude of non-study cigarette use is common for VLN cigarettes at different nicotine levels and across studies using different VLN cigarettes. Thus, understanding the extent of under-reporting of non-study cigarettes is an important consideration in interpreting the results. Using the method described here, the number of estimated under-reported non-study CPD exceeds the reduction in self-reported CPD after switching to VLN cigarettes for every group in all five studies assessed. Such high levels of non-compliance with non-study cigarette use, in both the prevalence and the magnitude, will 
likely impact other outcome measures including, craving, withdrawal, and dependence. In planning future ambulatory VLN cigarette switching studies, researchers should consider incorporating specific biomarkers that would enable objective assessment of both the prevalence and the magnitude of non-study cigarette use.

\section{Declarations}

\section{Abbreviations}

ANPRM: advance notice of proposed rulemaking; BOE: biomarker of exposure; CI: Canadian Intensive; CNC: conventional nicotine cigarette; CPD: cigarettes per day; ISO: International Organization for Standardization; NNAL: 4-(Methylnitrosamino)-1-(3-pyridyl)-1-butanol; NNK: 4-(methylnitrosamino)-1-(3-pyridyl)-1-butanone; TNE: nicotine equivalent; UB: usual brand; VLN: very low nicotine

\section{Acknowledgements}

The authors would like to thank Mike Morton for helpful discussions on formulating the initial equation to describe the relationship between BOE, Yield and CPD; Nghia Nguyen and Samuel Sparks for technical assistance in data analyses.

\section{Competing interests}

All authors are employed by Altria Client Services LLC and declare that they have no additional competing interests.

\section{Funding}

This work was supported by Altria Client Services LLC.

\section{Authors' contributions}

Mingda Zhang conceived the method and drafted the manuscript. Jeffery Edmiston, George Karles, and Donna Smith participated in the method development and data interpretation. All authors critically revised and approved the final manuscript for publication.

\section{Availability of data and material}

All data generated or analyzed during this study are included in this published article and its supplementary information files.

\section{Ethics approval and consent to participate}

Not applicable.

\section{Consent for publication}

Not applicable.

\section{References}

1. U. S. Food and Drug Administration: Tobacco Product Standard for Nicotine Level of Combusted Cigarettes. In., vol. 1130. 21 CFR; 2018: 11818-11843. 
2. Donny EC, Denlinger RL, Tidey JW, Koopmeiners JS, Benowitz NL, Vandrey RG, al'Absi M, Carmella SG, Cinciripini PM, Dermody SS et al: Randomized Trial of ReducedNicotine Standards for Cigarettes. The New England journal of medicine 2015, 373(14):1340-1349.

3. Benowitz NL, Nardone N, Hatsukami DK, Donny EC: Biochemical estimation of noncompliance with smoking of very low nicotine content cigarettes. Cancer Epidemiol Biomarkers Prev 2015, 24(2):331-335.

4. Nardone N, Donny EC, Hatsukami DK, Koopmeiners JS, Murphy SE, Strasser AA, Tidey JW, Vandrey R, Benowitz NL: Estimations and predictors of non-compliance in switchers to reduced nicotine content cigarettes. Addiction (Abingdon, England) 2016, 111(12):2208-2216.

5. Goldstein JA, Goldstein LK: Randomized Trial of Reduced-Nicotine Standards for Cigarettes. The New England journal of medicine 2016, 374(4):395-396.

6. Foulds J, Hobkirk A, Wasserman E, Richie J, Veldheer S, Krebs NM, Reinhart L, Muscat J: Estimation of compliance with exclusive smoking of very low nicotine content cigarettes using plasma cotinine. Preventive medicine 2018, 117:24-29.

7. Dermody SS, Donny EC, Hertsgaard LA, Hatsukami DK: Greater reductions in nicotine exposure while smoking very low nicotine content cigarettes predict smoking cessation. Tobacco control 2015, 24(6):536-539.

8. Hatsukami DK, Donny EC, Koopmeiners JS, Benowitz NL: Compensatory smoking from gradual and immediate reduction in cigarette nicotine content. Cancer epidemiology, biomarkers \& prevention : a publication of the American Association for Cancer Research, cosponsored by the American Society of Preventive Oncology 2015, 24(2):472-476.

9. Hammond D, O'Connor RJ: Reduced nicotine cigarettes: smoking behavior and biomarkers of exposure among smokers not intending to quit. Cancer epidemiology, biomarkers \& prevention : a publication of the American Association for Cancer Research, cosponsored by the American Society of Preventive Oncology 2014, 23(10):2032-2040.

10. Mercincavage M, Souprountchouk V, Tang KZ, Dumont RL, Wileyto EP, Carmella SG, Hecht SS, Strasser AA: A Randomized Controlled Trial of Progressively Reduced Nicotine Content Cigarettes on Smoking Behaviors, Biomarkers of Exposure, and Subjective Ratings. Cancer epidemiology, biomarkers \& prevention : a publication of the American Association for Cancer Research, cosponsored by the American Society of Preventive Oncology 2016, 25(7):1125-1133.

11. Hatsukami DK, Luo X, Jensen JA, al'Absi M, Allen SS, Carmella SG, Chen M, Cinciripini PM, Denlinger-Apte R, Drobes DJ et al: Effect of Immediate vs Gradual Reduction in Nicotine Content of Cigarettes on Biomarkers of Smoke Exposure: A Randomized Clinical Trial. Jama 2018, 320(9):880-891.

12. Denlinger RL, Smith TT, Murphy SE, Koopmeiners JS, Benowitz NL, Hatsukami DK, Pacek LR, Colino C, Cwalina SN, Donny EC: Nicotine and Anatabine Exposure from Very Low Nicotine Content Cigarettes. Tobacco regulatory science 2016, 2(2):186-203.

13. Nicotine Research Cigarettes Drug Supply Program [ https://www.drugabuse.gov/nicotine-research-cigarette-drug-supply-program] 
14. Cassidy RN, Colby SM, Tidey JW, Jackson KM, Cioe PA, Krishnan-Sarin S, Hatsukami D: Adolescent smokers' response to reducing the nicotine content of cigarettes: Acute effects on withdrawal symptoms and subjective evaluations. Drug and alcohol dependence 2018, 188:153-160.

15. Faulkner P, Ghahremani DG, Tyndale RF, Cox CM, Kazanjian AS, Paterson N, Lotfipour S, Hellemann GS, Petersen N, Vigil C et al: Reduced-Nicotine Cigarettes in Young Smokers: Impact of Nicotine Metabolism on Nicotine Dose Effects. Neuropsychopharmacology : official publication of the American College of Neuropsychopharmacology 2017, 42(8):1610-1618.

16. Higgins ST, Heil SH, Sigmon SC, Tidey JW, Gaalema DE, Hughes JR, Stitzer ML, Durand H, Bunn JY, Priest JS et al: Addiction Potential of Cigarettes With Reduced Nicotine Content in Populations With Psychiatric Disorders and Other Vulnerabilities to Tobacco Addiction. JAMA psychiatry 2017, 74(10):1056-1064.

17. Perkins KA, Kunkle N, Karelitz JL: Threshold dose for behavioral discrimination of cigarette nicotine content in menthol vs. non-menthol smokers.

Psychopharmacology 2017, 234(8):1255-1265.

18. Perkins KA, Kunkle N, Karelitz JL, Michael VC, Donny EC: Threshold dose for discrimination of nicotine via cigarette smoking. Psychopharmacology 2016, 233(12):2309-2317.

19. Shiffman S, Kurland BF, Scholl SM, Mao JM: Nondaily Smokers' Changes in Cigarette Consumption With Very Low-Nicotine-Content Cigarettes: A Randomized Double-blind Clinical Trial. JAMA psychiatry 2018.

20. Jarvis MJ, Russell MA, Benowitz NL, Feyerabend C: Elimination of cotinine from body fluids: implications for noninvasive measurement of tobacco smoke exposure. American journal of public health 1988, 78(6):696-698.

21. Benowitz NL, Jacob P, 3rd: Metabolism of nicotine to cotinine studied by a dual stable isotope method. Clinical pharmacology and therapeutics 1994, 56(5):483-493.

22. Benowitz NL, Hall SM, Stewart S, Wilson M, Dempsey D, Jacob P, 3rd: Nicotine and carcinogen exposure with smoking of progressively reduced nicotine content cigarette. Cancer epidemiology, biomarkers \& prevention : a publication of the American Association for Cancer Research, cosponsored by the American Society of Preventive Oncology 2007, 16(11):2479-2485.

23. Mercincavage M, Smyth JM, Strasser AA, Branstetter SA: Reduced Nicotine Content Expectancies Affect Initial Responses to Smoking. Tobacco regulatory science 2016 , $2(4): 309-316$.

24. Benowitz NL, Nardone N, Dains KM, Hall SM, Stewart S, Dempsey D, Jacob P, 3rd: Effect of reducing the nicotine content of cigarettes on cigarette smoking behavior and tobacco smoke toxicant exposure: 2-year follow up. Addiction (Abingdon, England) 2015, 110(10):1667-1675.

25. Ding YS, Richter P, Hearn B, Zhang L, Bravo R, Yan X, Perez JJ, Chan M, Hughes J, Chen P et al: Chemical Characterization of Mainstream Smoke from SPECTRUM Variable Nicotine Research Cigarettes. Tobacco regulatory science 2017, 3(1):81-94.

26. Richter P, Steven PR, Bravo R, Lisko JG, Damian M, Gonzalez-Jimenez N, Gray N, Keong LM, Kimbrell JB, Kuklenyik P et al: Characterization of SPECTRUM Variable 
Nicotine Research Cigarettes. Tobacco regulatory science 2016, 2(2):94-105.

27. Mendes P, Liang Q, Frost-Pineda K, Munjal S, Walk RA, Roethig HJ: The relationship between smoking machine derived tar yields and biomarkers of exposure in adult cigarette smokers in the US. Regul Toxicol Pharmacol 2009, 55(1):17-27.

28. Theophilus EH, Coggins CR, Chen P, Schmidt E, Borgerding MF: Magnitudes of biomarker reductions in response to controlled reductions in cigarettes smoked per day: a one-week clinical confinement study. Regul Toxicol Pharmacol 2015, 71(2):225-234.

29. Jarvis MJ, Foulds J, Feyerabend C: Exposure to passive smoking among bar staff. British journal of addiction 1992, 87(1):111-113.

30. Roethig HJ, Munjal S, Feng S, Liang Q, Sarkar M, Walk RA, Mendes PE: Population estimates for biomarkers of exposure to cigarette smoke in adult U.S. cigarette smokers. Nicotine \& tobacco research : official journal of the Society for Research on Nicotine and Tobacco 2009, $11(10): 1216-1225$.

31. Hatsukami DK, Luo X, Dick L, Kangkum M, Allen SS, Murphy SE, Hecht SS, Shields PG, al'Absi M: Reduced nicotine content cigarettes and use of alternative nicotine products: exploratory trial. Addiction (Abingdon, England) 2016, 112(1):156-167.

\section{Figures}




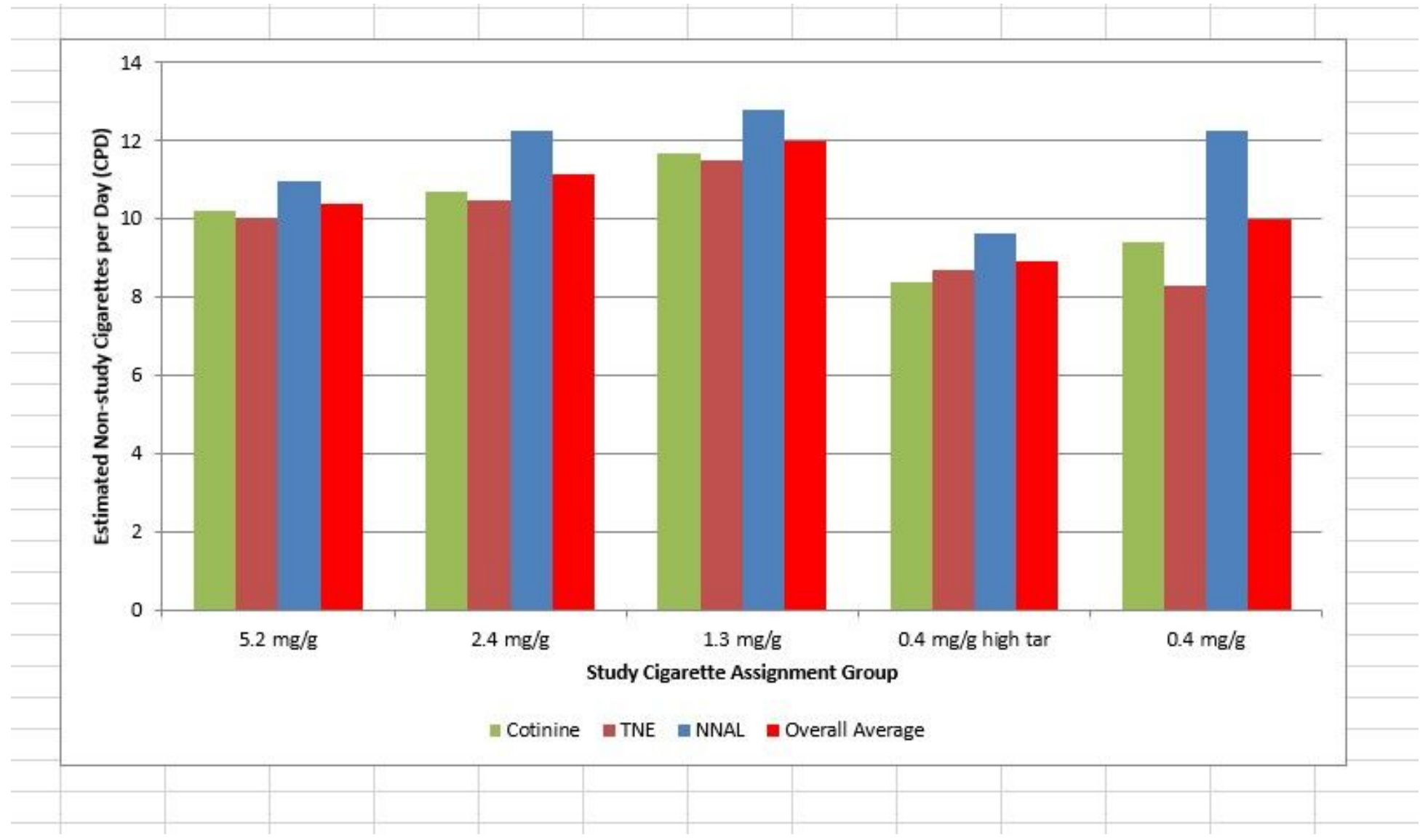

\section{Figure 1}

Estimated Number of Non-study CPD Estimated number of non-study CPD for each VLN cigarette group in Donny et al. [2], using $15.8 \mathrm{mg} / \mathrm{g}$ CNC cigarette as the reference. The first three columns in each group were average values based on cotinine, TNE and NNAL. The last column for each VLN cigarette group represents the mean of the preceding three estimates.

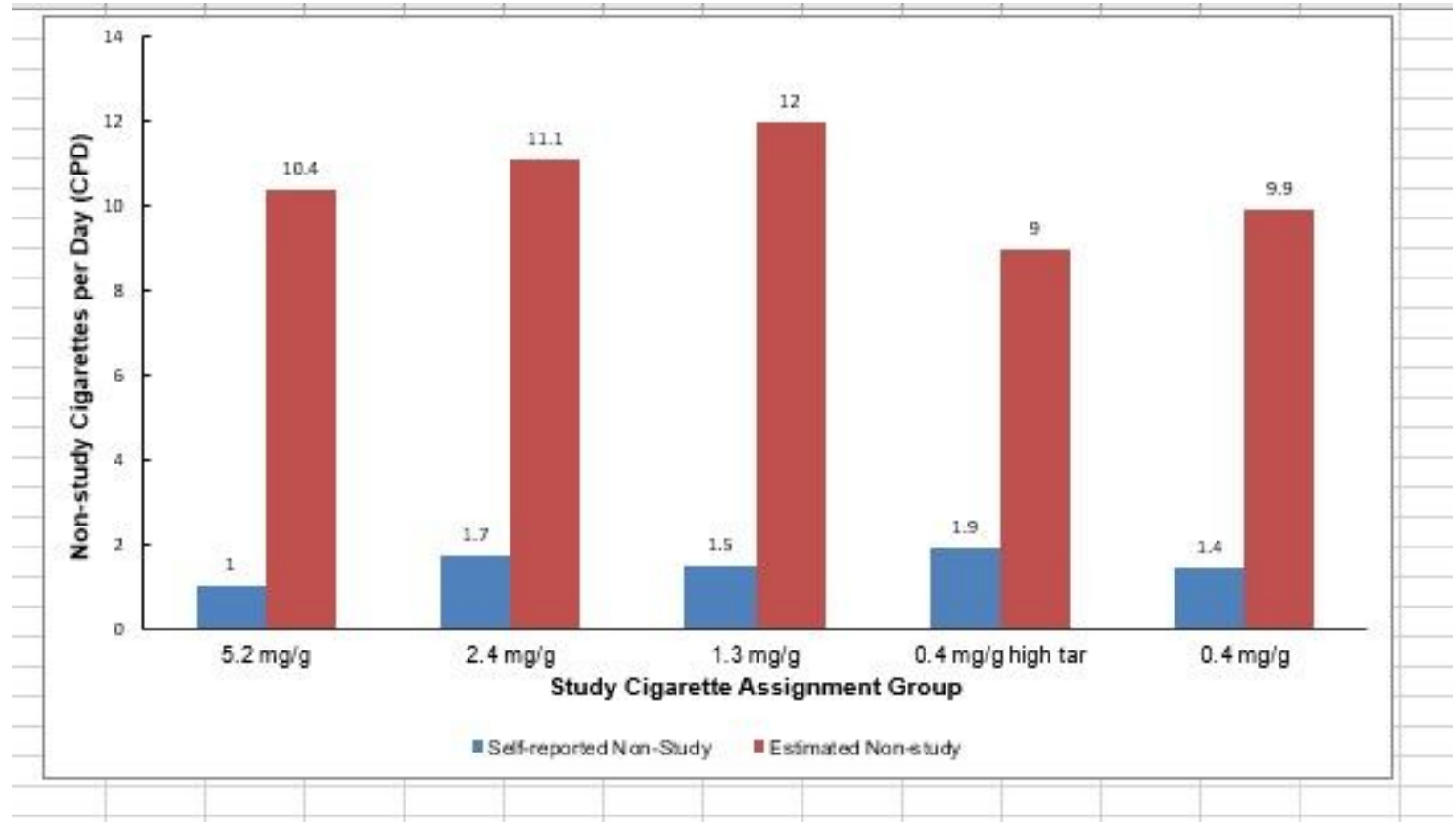


Figure 2

Average Self-reported and Estimated Number of Non-study CPD Average self-reported and estimated number of non-study CPD for each VLN cigarette group in Donny et al. [2], with $15.8 \mathrm{mg} / \mathrm{g} \mathrm{CNC}$ cigarettes as the reference.

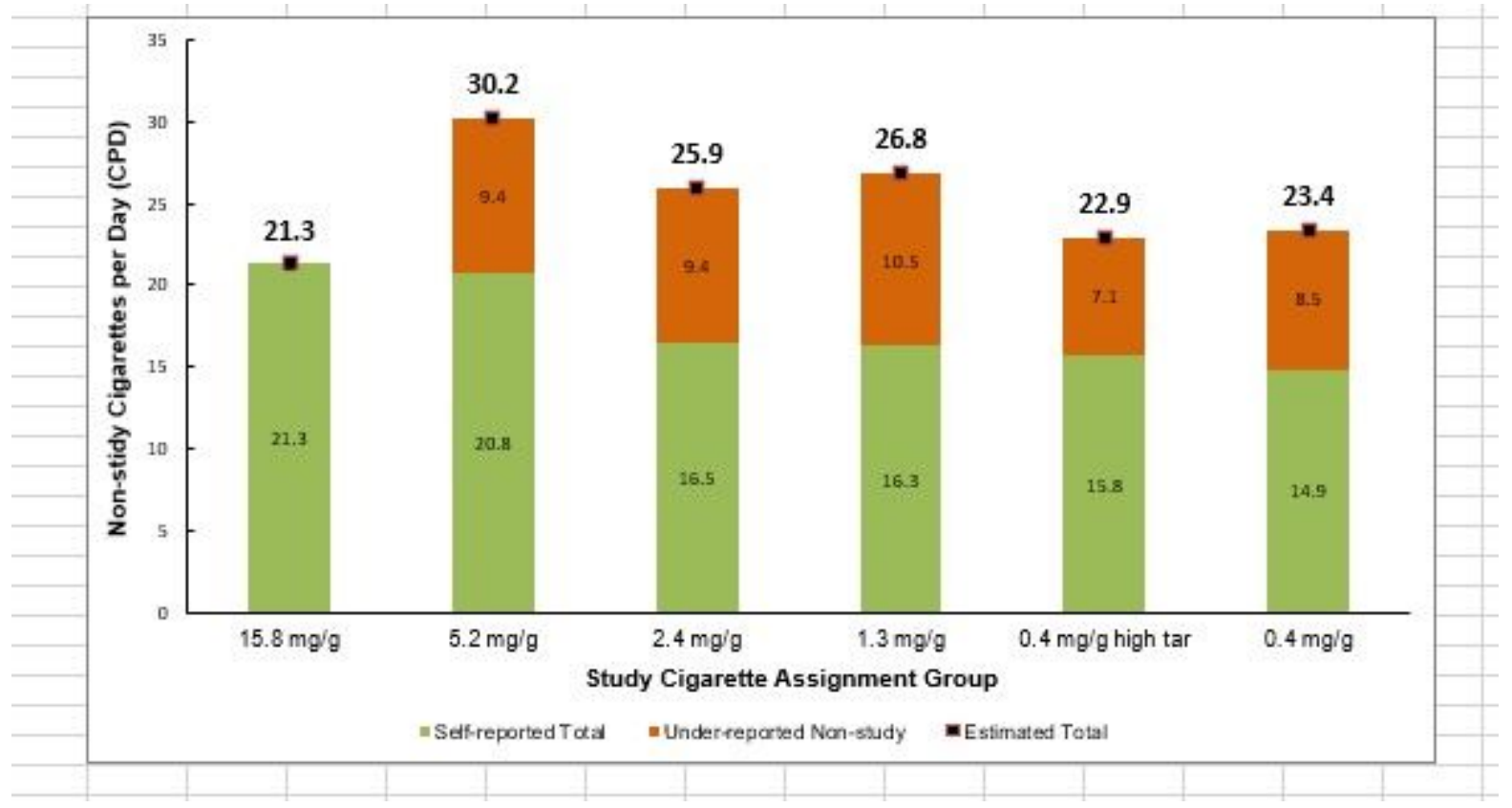

\section{Figure 3}

Average Self-reported Total, Estimated Under-reported, and Estimated Total CPD Average self-reported total, estimated under-reported, and estimated total CPD for each VLN cigarette group in Donny et al. [2], with $15.8 \mathrm{mg} / \mathrm{g}$ CNC cigarettes as reference. 

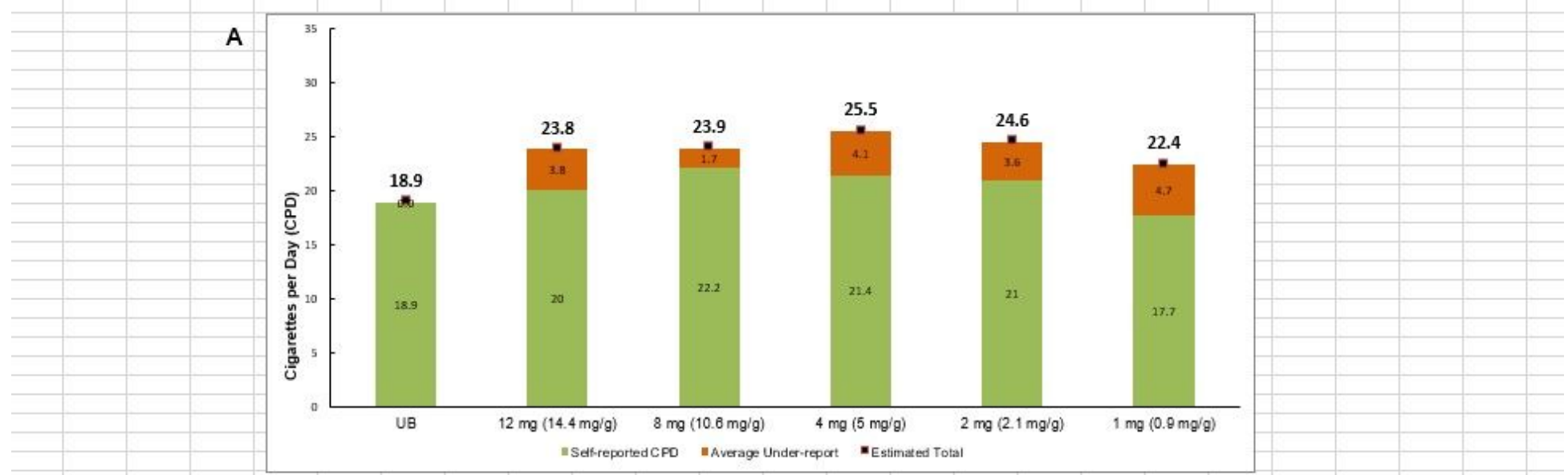

B
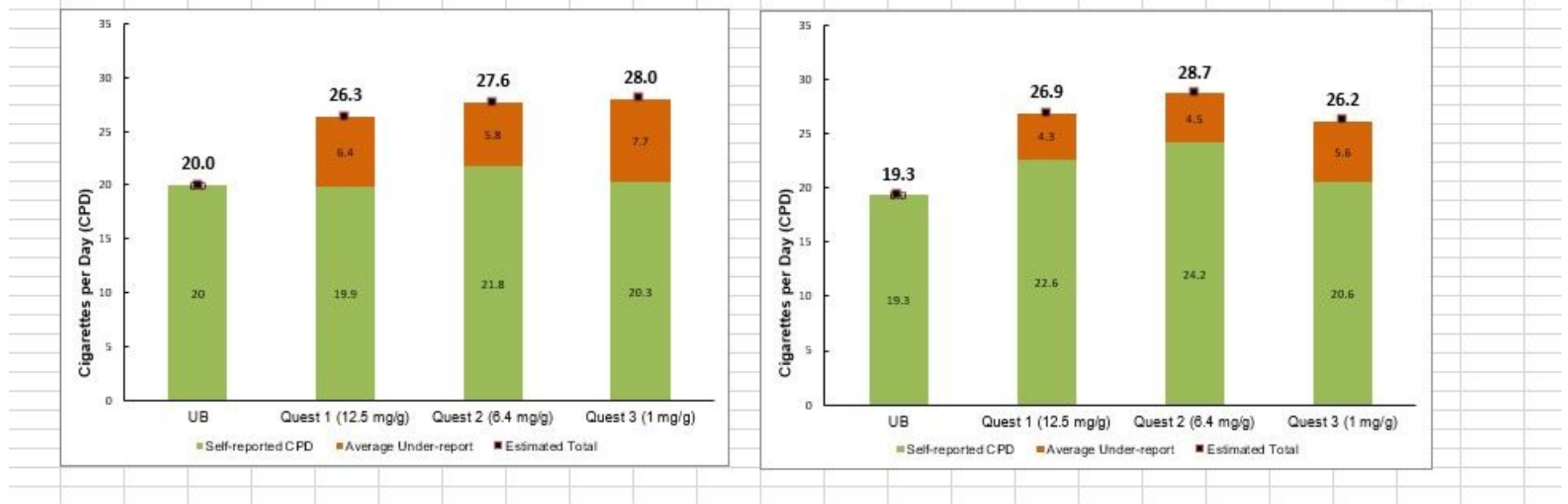

Figure 4

Self-reported, Estimated Under-reported and Estimated Total CPD Self-reported, estimated under-reported and estimated total CPD based on nicotine exposure using participants' UB cigarettes as reference. A: average estimates based on the nicotine content in filler and FTC smoke nicotine yield compared to plasma cotinine from Benowitz et al. [22]; B: estimates based on FTC nicotine yield compared to urinary cotinine from Hammond and O'Connor [9]; C: average estimates based on FTC nicotine yield compared to urinary cotinine and urinary nicotine from Mercincavage et al. [10], all with UB cigarettes as the reference. The numbers in the parentheses next to the product name at the bottom indicate the nicotine content per gram of tobacco filler of the cigarettes used in the studies.

\section{Supplementary Files}

This is a list of supplementary files associated with this preprint. Click to download.

- NonstudyCPDEstimateMethodBMCAdditionalFiles.docx 